\title{
Toxicidade reprodutiva do extrato hidroalcóolico de Samanea tubulosa Benth em ratas wistar
}

SALES, P.A.B ${ }^{\text {**; }}$ OLIVEIRA, J.M.G.'; MOURA, E.R.'; SOUSA, M.R.S.C.1; SILVA, I.R. '; COSTA, T.N.'; COSTA, A.P.R. ${ }^{1}$

'Universidade Federal do Piauí, Campus Universitário Ministro Petrônio Portela, Campus Socopo-CCA, Teresina, PI, CEP 64049-550. *Autor para correspondência: pauloalexbezerra@hotmail.com

RESUMO: O objetivo deste trabalho foi avaliar possíveis efeitos do extrato das vargens de bordão-de-velho (Samanea tubulosa benth) sobre a reprodução em ratas Wistar. O extrato foi preparado a partir da secagem e trituração das vagens, seguida por maceração com etanol e, posteriormente, concentrado em rotavapor. Ratas Wistar gestantes foram tratadas com 75 mg. $100 \mathrm{~g}^{-1}$ de peso corporal de extrato de $S$. tubulosa e o grupo controle recebeu $1 \mathrm{ml} / 100 \mathrm{~g}^{-1} \mathrm{de}$ peso corporal de solução salina por gavagem durante 19 dias. Após eutanásia no $20^{\circ}$ dia, as seguintes variáveis foram analisadas: peso corporal fetal, peso dos fetos e placentas, número de corpos lúteos e de reabsorções, implantação, fetos vivos e mortos. O extrato de vagens de S. tubulosa apresenta toxicidade para o feto causando malformações, reabsorções fetais e diminuição pronunciada do peso ao nascer. Contudo, o extrato de vagens de $S$. tubulosa não influencia no número de corpos lúteos e número de implantações.

Palavras-chave: Plantas tóxicas, malformação, potencial tóxico

\begin{abstract}
Reproductive toxicity study in Wistar rats treated with hydroalcoholic extract of the pods of bordão-de-velho (Samanea tubulosa Benth). The aim of this study was to evaluate possible effects of pods from bordão-de-velho (Samanea tubulosa benth) extract on the reproduction of female rats. The extract was prepared by maceration wtith ethanol of dried and crushed pods, then it was concentrated in rotary evaporator. The pregnant Wistar rats were treated with a dose of $75 \mathrm{mg} .100 \mathrm{~g} \mathrm{~g}^{-1}$ of body weight of Samanea tubulosa extract, by gavage , whereas the control group received $1 \mathrm{ml}^{1} 100 \mathrm{~g}^{-1}$ of saline by the same route for 19 days. After the euthanasia of the animals at day 20th,the following variables were analyzed: fetal body weight, weight of fetuses and placentas, number of corpora lutea, resorption points, live and dead fetuses and number of embryonic implantations. The extract from $S$. tubulosa pods, at the dose studied, showed toxicity causing fetal abnormalities, fetal resorption and pronounced decrease in the weight of offspring at birth. On the other hand, , the treatment with extract of $S$. tubulosa pods had no influence over corpora lutea and implantation numbers.
\end{abstract}

Key words:Toxic plants, malformation, potential toxic.

\section{INTRODUÇÃO}

O Brasil possui uma vasta diversidade biológica, contando com uma rica flora, despertando interesses de comunidades científicas para o estudo, conservação e utilização racional destes recursos. Diante desta grande diversidade, muitas plantas são utilizadas para fins medicinais, oferecendo uma contribuição ao desenvolvimento da ciência, a partir de conhecimentos e práticas de saúde de caráter empírico (Pereira et al., 2004; Souza \& Felfili, 2006).

Contudo, as plantas possuem substâncias ativas e, muitas vezes, o efeito tóxico sobre o organismo é desconhecido ou ignorado pelos usuários. Daí a importância do conhecimento sobre o consumo de plantas para fins terapêuticos para uso seguro, eficaz e consciente (Arruda et al., 2013)

A Samanea tubulosa Benth, espécie da família Leguminosae, é uma espécie nativa da região do pantanal e amazônica, sendo uma planta cultivada com propósitos ornamentais e na arborização rural. $O$ fruto doce dessa espécie é muito procurado pelo gado e as ramas são forrageiras, com alto teor de proteína bruta. Em 
vários países faz-se uma farinha com os frutos, que é um alimento excelente para as vacas, cabras e galinhas. Para humanos, as vagens são comestíveis e a polpa é doce com sabor de alcaçuz, com $25 \%$ de açúcar, que deve ser conservada seca e cristalizada. Os frutos fermentam e dão álcool com rendimento, aproximadamente, de 11,5 L para cada $100 \mathrm{~kg}$ de frutos. Com ele, pode-se também produzir aguardente, conhecida como "aguardentede-saman" semelhante ao kirsch (Embrapa, 2007; Lima et al., 2009).

Esta espécie vegetal possui diversos nomes vulgares distintos por Unidades da Federação: em Alagoas, no Maranhão, na Paraíba, em Pernambuco e em Sergipe, bordão-de-velho; na Bahia, samaneiro e sete-cascas; no Ceará, barba-de-velho e bordãode-velho; em Mato Grosso e em Mato Grosso do Sul, abobreira, alfarobo, farinha-seca, feijão-cru, ingáde-pobre, pau-de-cangaia e setecascas (Embrapa, 2007; Lorenzi \& Souza, 2011).

Estudo realizado com extrato metanólico das folhas de $S$. tubulosa, verificou-se a presença de compostos bioativos como: alcalóides, triterpenóides e compostos fenólicos como taninos, flavonóides e cumarinas. Também foi observada uma atividade antioxidante que pode estar relacionada com a presença de compostos polifenólicos, como os flavonóides, que são capazes de proteger contra várias doenças crônicas (Lima et al., 2009).

A S. tubulosa foi relatada por produtores rurais como tóxica para bovinos por causar abortos, porém sem comprovação cientifica (Costa et al., 2011). Cruz (2006) descreveu seu uso na medicina popular para o tratamento de infecções cutâneas, inflamações gástricas e infestações parasitárias.

Nesse contexto, devido à importância de pesquisas envolvendo plantas, nosso objetivo foi avaliar possíveis efeitos do extrato de vagens Samanea tubulosa sobre a reprodução em ratas Wistar e assim contribuir para a elucidação da toxicidade reprodutiva desta espécie.

\section{MATERIAL E MÉTODO}

Foram utilizadas 16 ratas adultas da linhagem Wistar, pesando entre 180 a 250 gramas, criadas e mantidas no Biotério do Centro de Ciências Agrárias da Universidade Federal do Piauí. Os animais foram mantidos em gaiolas, tendo livre acesso a água e ração.

O extrato foi preparado no Laboratório de Fisiologia e Farmacologia da Universidade Federal do Piauí. Primeiramente coletaram-se as vagens de Samanea tubulosa Benth de árvores da região rural de Teresina-PI. Uma exsicata $n^{\circ} 27.261$ encontra-se depositada no acervo do Herbário Graziela Barroso (TEPB) da Universidade Federal do Piauí (UFPI). Secou-se as vagens em estufa com circulação mecânica modificada a $45^{\circ} \mathrm{C}$ durante 5 dias, posteriormente foi triturada em forrageira para o fracionamento das vagens, sendo depois levada ao moinho elétrico para se obter uma granulometria de tamanho adequado para a preparação do extrato. Usou-se $300 \mathrm{~g}$ de pó de $S$. tubulosa para cada 1 litro de álcool a $70 \%$, fez-se 5 substituições de álcool para obtenção do extrato. O extrato foi concentrado em Evaporador Rotativo a uma temperatura de $50^{\circ} \mathrm{C}$. A solução foi preparada no laboratório de fisiologia e farmacologia chegando à concentração desejada de $75 \mathrm{mg} / \mathrm{ml}$ (Branco Neto et al., 2006).

O esfregaço vaginal foi realizado diariamente sempre no período de 8 as 9 horas da manhã, para verificar qual fase do ciclo estral se apresentava cada animal, aquela constatada em proestro era colocada na presença de um macho, no dia seguinte fazia-se novamente um esfregaço vaginal para detecção de espermatozóides, considerando assim o primeiro dia de gestação.

Foram divididos em dois grupos de 8 animais: Grupo 1 (controle) recebeu solução salina por gavagem $\left(1 \mathrm{~mL} / 100 \mathrm{~g} \mathrm{~g}^{-1}\right)$ do $1^{\circ}$ ao $19^{\circ}$ dia de gestação e o Grupo 2 (extrato) recebia extrato (75 $\mathrm{mg} / \mathrm{ml}$ ), na dose: de $1 \mathrm{~mL} / 100 \mathrm{~g}$ do $1^{\circ}$ ao $19^{\circ}$ dia de gestação, todos administrados com o auxilio de uma cânula gástrica adequada aos animais.

Ao $20^{\circ}$ dia de gestação as ratas foram eutanasiadas (xilazina+ketamina) e realizadas as cesarianas, removeu-se o útero gravídico para a obtenção dos seguintes dados: números de fetos vivos e mortos pela avaliação dos batimentos cardíacos e frequência respiratória, avaliação do peso individual e da ninhada, número de sítios de implantações, reabsorções e corpos lúteos, pesagem das placentas de todos os fetos e exame macroscópico (malformação e reabsorção fetal).

Para o levantamento desses dados fez-se uso de equipamentos como balança de precisão lupa e material cirúrgico adequado. $O$ este estudo foi aprovado pelo Comitê de Ética em Pesquisa da Universidade Federal do Piauí com parecer $\mathrm{n}^{\circ}$ 033/09.

O teste estatístico realizado foi o " $\mathrm{t}$ " de Student com nível de significância dos testes $=0,05$, usando o prism 5.0.

\section{RESULTADOS E DISCUSSÃO}

A pesquisa realizada apresentou dados significativos quanto a alguns parâmetros avaliados, por exemplo, na comparação entre os pesos fetais do grupo controle e extrato de $S$. tubulosa (Figura 1 e Tabela 1). Já a respeito ao peso da placenta, quantidade de corpos lúteos, taxa de implantação não foram evidenciados diferenças estatísticas 


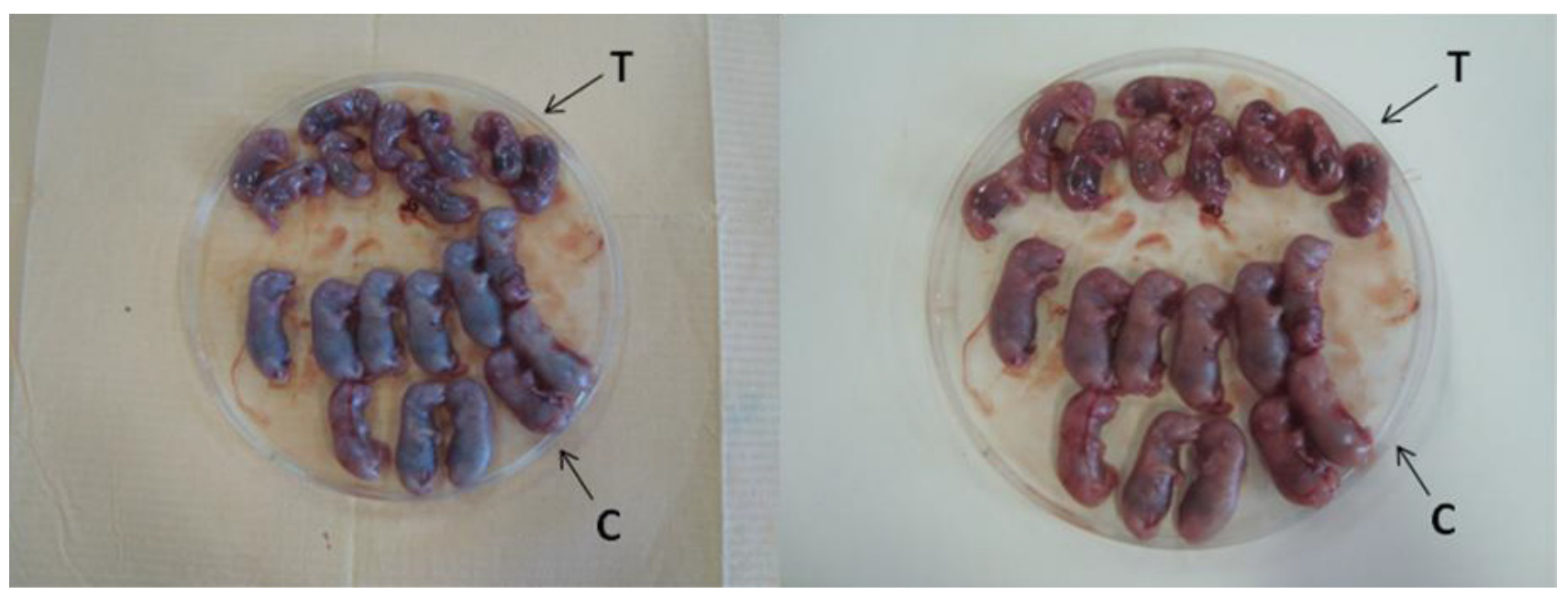

FIGURA 1. Diferença no peso dos fetos em ratas submetidas aos tratamentos com Extrato de Samanea tubulosa (T) e com Solução Salina(C).

TABELA 1. Peso corporal(g) dos fetos ao nascer e placentário do grupo controle e extrato.

\begin{tabular}{lcc}
\hline Parâmetro & Controle & Extrato \\
\hline Peso dos fetos & $3.36 \pm 1.288$ & $1.85 \pm 0.698$ \\
\hline Peso placentário & $0.628 \pm 0.083$ & $0.375 \pm 0.7440$ \\
\hline
\end{tabular}

Resultados expressos em média \pm desvio padrão $(p>0,05)$ pelo teste "t" de Student. *Diferença significativa em relação ao grupo-controle $(\mathrm{P}<0,05)$

TABELA 2. Número de corpos lúteos e implantações encontradas no grupo controle e extrato.

\begin{tabular}{lcc}
\hline \multicolumn{1}{c}{ Parâmetro } & Controle & Extrato \\
\cline { 2 - 3 } $\mathrm{N}^{\circ}$ de Implantação & $8.38 \pm 1.51$ & $8.88 \pm 2.36$ \\
\hline & $9.63 \pm 1.69$ & $11.75 \pm 3.28$ \\
$\mathrm{~N}^{\circ}$ de Corpo Lúteo & \\
\hline $\begin{array}{l}\text { Resultados expressos em média } \pm \text { desvio padrão }(p>0,05) \text { pelo teste "t" de Student. *Diferença significativa em relação ao grupo-controle } \\
(\mathrm{P}<0,05)\end{array}$
\end{tabular}
$(\mathrm{P}<0,05)$

(Tabela 2). Dentre os resultados encontrados o que chama bastante atenção foi a presença de malformações fetais, assim como reabsorções fetais no grupo tratado com extrato de S. tubulosa (Figura 2 e 3 ). Alguns estudos corroboram com os resultados obtidos mostrando o que a $S$. tubulosa poderia ter um potencial tóxico na gestação, como foi relatado um caso de abortamento em uma vaca no município de Filadélfia em Tocantins (Costa, 2009).

Com base em estudos fitoquímicos (Lima et al., 2009) foi revelado à presença de alcalóides, triterpenóides e compostos fenólicos como taninos, flavonóides e cumarinas em extrato de $S$. tubulosa. Com esse estudo verificamos que tanto o tanino como as cumarinas podem ser possíveis causadores das malformações fetais, reabsorção fetal e do baixo ganho de peso do grupo tratado com S. tubulosa, necessitando de estudos posteriores para afirmar contundentemente tal colocação.

A toxicidade causada pelo extrato de $S$. tubulosa não deixam dúvidas de seu poder tóxico aos animais e possivelmente a humanos, onde o seu consumo têm que ser reavaliado, pois já foi relatado seu uso como fitoterápico e na alimentação animal 

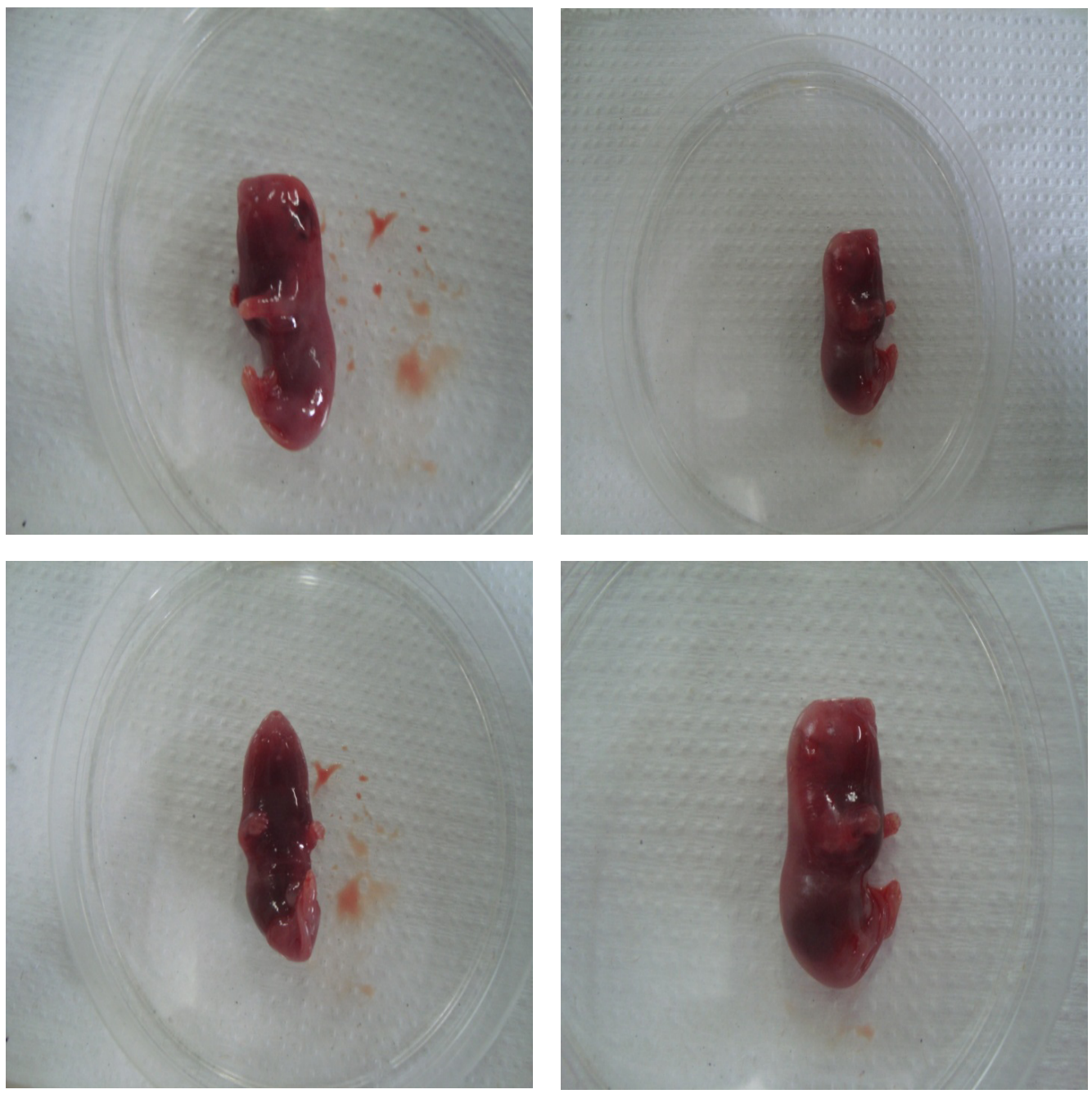

FIGURA 2. Feto do grupo de ratas tratadas com extrato hidroalcoólico de Samanea tubulosa apresentando má formação de ordem morfológica, distinção imprecisa entre cabeça e pescoço. Fonte: Paulo Alex Bezerra Sales, 2010.

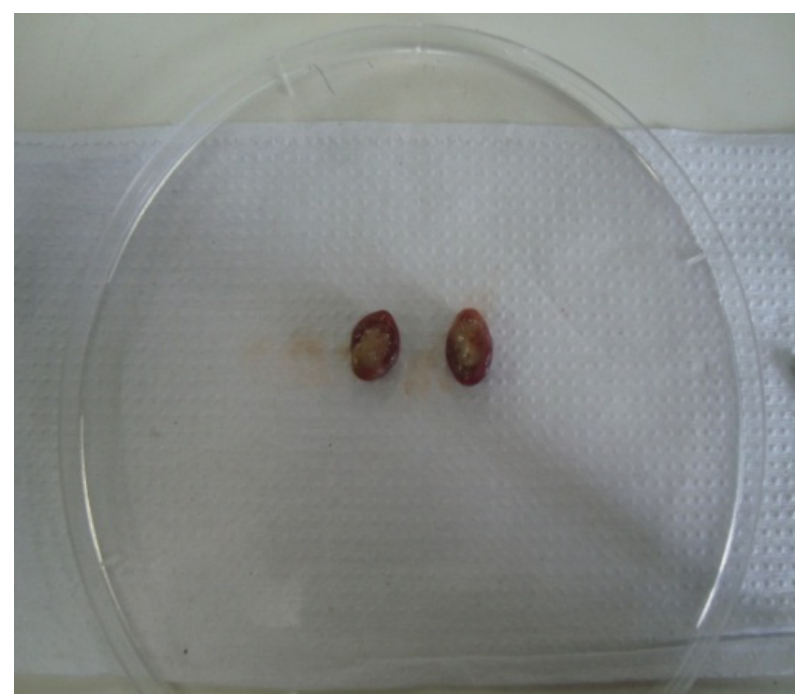

FIGURA 3. Presença de placentas sem feto, indicativas de reabsorções fetais no grupo tratado com Samanea tubulosa. Fonte: Paulo Alex Bezerra Sales, 2010.
(Cruz, 2006; Embrapa, 2007).

A média e o desvio padrão dos pesos placentários não houve diferenças significativas, onde o peso dos fetos ao nascer foi observado diferença significativa entre o grupo tratado e controle (Tabela 1). A quantidade de corpos lúteos e taxa de implantação não foram evidenciadas diferenças estatísticas (Tabela 2). Não foram encontradas diferenças significativas em relação ao número de fetos vivos e fetos mortos entre os grupos estudados (Tabela 3 ).

Baseados nos resultados obtidos sugerese que o extrato vagens de Samanea tubulosa apresentam toxicidade reprodutiva, uma vez que os fetos apresentaram malformações, reabsorções fetais e diminuição pronunciada do peso ao nascer. Contudo o extrato vargens de $S$. tubulosa não influencia em relação ao número de corpos lúteos e número de implantações. 
TABELA 3. Número de fetos nascidos vivos e mortos do grupo controle e extrato

\begin{tabular}{lcc}
\hline \multicolumn{1}{c}{ Parâmetro } & Controle & Extrato \\
\hline $\mathrm{N}^{\circ}$ Fetos vivo & $7.88 \pm 1.73$ & $7.13 \pm 3.40$ \\
\hline $\mathrm{N}^{\circ}$ Fetos mortos & $0.250 \pm 0.707$ & $1.375 \pm 1.685$
\end{tabular}

Resultados expressos em média \pm desvio padrão $(p>0,05)$ pelo teste " $t$ " de Student . *Diferença significativa em relação ao grupo-controle $(P<0,05)$

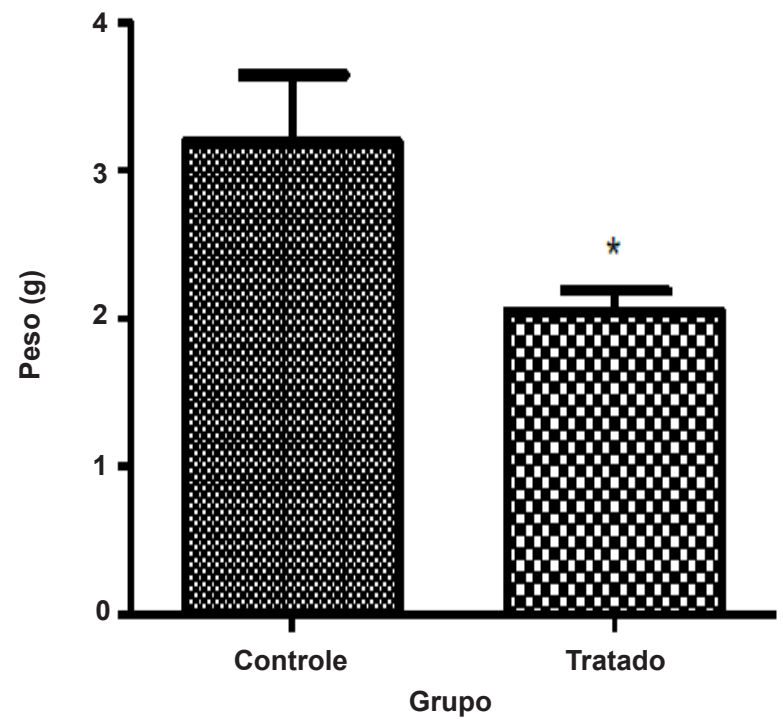

FIGURA 4. Pesos dos fetos de ratas submetidas aos tratamentos com solução salina e com extrato hidroalcoólico de Samanea tubulosa. *Diferença significativa em relação ao grupo-controle $(P<0,05)$

\section{AGRADECIMENTO}

À CAPES - Centro de aperfeiçoamento de Pessoal de nível Superior, pelo suporte financeiro; a Universidade Federal do Piauí - UFPI pela oportunidade de desenvolver esse projeto e a todos os meus colaboradores.

\section{REFERÊNCIAS}

ARRUDA, J.T. et al. Efeito do extrato aquoso de camomila (Chamomilla recutita L.) na prenhez de ratas e no desenvolvimento dos filhotes. Revista Brasileira
Plantas Medicinais, v.15, n.1, p.66-71, 2013.

BRANCO NETO, M.L.C. et al. Avaliação do extrato hidroalcoólico de Aroeira (Schinus terebinthifolius Raddi) no processo de cicatrização de feridas em pele de ratos. Acta Cirúrgica Brasileira, v.21, n.2, p.17-22, 2006.

COSTA, A.M.D. et al. Plantas tóxicas de interesse pecuário em região de ecótono amazônia e cerrado. parte araguaína, norte do Tocantins. Acta Veterinaria Brasilica, v.5, n.3, p.317-324, 2011.

COSTA, A.M.D. Plantas tóxicas de interesse pecuário nas microrregiões de Araguaína e Bico do papagaio, norte do Tocantins. 2009. 104p. Dissertação (Mestrado em Ciência Animal Tropical) Universidade Federal do Tocantins, Araguaína.

CRUZ, R. B. Caracterização Estrutural e Atividade Biológica de Constituintes Isolados de Samanea tubulosa. In: JORNADA GIULIO MASSARANI DE INICIAÇÃO CIENTÍFICA, ARTÍSTICA E CULTURAL, XXVII, 2006, Rio de Janeiro. Anais. Rio de Janeiro: UFRJ- Centro de Ciências da Saúde, 2006, p.149-150.

EMBRAPA. Bordão-de-Velho - Samanea tubulosa. Colombo, PR: Embrapa Florestas, 2007. 6p. (Embrapa Florestas. Circular Técnica, 132). Disponível em: $<$ http://ibflorestas.org.br/en/pdf/category/1-pdf. html?download=4\%3Aaspectos-gerais-de-arvore-dachuva. >. Acesso em: 23 jun. 2013.

LIMA, I.V. et al., Potencial químico e biológico de Samanea tubulosa. In: REUNIÃO ANUAL DA SOCIEDADE BRASILEIRA DE QUÍMICA, 32ª, 2009, Fortaleza. Anais. São Paulo: Sociedade Brasileira de Química (SBQ), 2009.

LORENZI, H.; SOUZA H.M. Plantas ornamentais no Brasil: arbustivas, herbáceas e trepadeiras. 4. ed. São Paulo: Plantarum, 2011. 1120p.

PEREIRA, R.C. et al. Plantas utilizadas como medicinais no município de Campos de Goytacazes - RJ. Revista Brasileira de Farmacognosia, v. 14, n.1, p.37-40, 2004.

SOUZA, C.D.; FELFILI, J. M. Uso de plantas medicinais na região de Alto Paraíso de Goiás, GO, Brasil. Acta Botanica Brasilica, v.20, n.1, p.135-142, 2006. 